\title{
Development of Fluorescence XAFS System in Soft X-Ray Region Toward Operando Conditions Using Polycapillary X-Ray Lens*
}

\author{
Mitsunori Honda, ${ }^{\dagger}$ Iwao Shimoyama, and Yuji Baba \\ Quantum Beam Science Center, Japan Atomic Energy Agency (JAEA), \\ 2-4, Shirakata, Tokai-mura, Naka-gun, Ibaraki 319-1195, Japan \\ Shinichi Suzuki \\ Quantum Beam Science Center, Japan Atomic Energy Agency (JAEA), \\ 2-4, Shirakata, Tokai-mura, Naka-gun, Ibaraki 319-1195, Japan, and \\ Fukushima Environmental Safety Center, Japan Atomic Energy Agency (JAEA), \\ 6-6, Sakae-Machi, Fukushima-shi, Fukushima 960-8031, Japan \\ Yoshihiro Okamoto \\ Quantum Beam Science Center, Japan Atomic Energy Agency (JAEA), \\ 2-4, Shirakata, Tokai-mura, Naka-gun, Ibaraki 319-1195, Japan \\ Tsuyoshi Yaita \\ Quantum Beam Science Center, Japan Atomic Energy Agency (JAEA), \\ 2-4, Shirakata, Tokai-mura, Naka-gun, Ibaraki 319-1195, Japan, and \\ Fukushima Environmental Safety Center, Japan Atomic Energy Agency (JAEA), \\ 6-6, Sakae-Machi, Fukushima-shi, Fukushima 960-8031, Japan \\ (Received 13 January 2015; Accepted 22 January 2016; Published 20 February 2016)
}

\begin{abstract}
We developed a fluorescence XAFS system toward operando conditions using soft x-ray radiation at KEK-PF BL-27A. X-ray adsorption fine structure (XAFS) analysis in the soft x-ray region is useful for elucidating molecular structures in both atmosphere and solutions. Particularly, light elements play an important role in many cases in this energy region. The attenuation of soft x-rays in a solution is large compared to that of hard x-rays. Thus, appropriate spectral statistics cannot be obtained in the soft x-ray region. Recently, using fluorescence XAFS measurement at the S K-edge $(2.4 \mathrm{keV})$, we found that biological molecules containing sulfur atoms adopt specific molecular structures under different $\mathrm{pH}$ conditions in a solution. However, the diameter of the beam of this beam line at BL-27A was large, leading to difficulties in uniformly irradiating only the sample surface. Therefore, it was necessary to uniformly irradiate samples using a small-area x-ray beam. To collimate the beam and improve its intensity, we installed an x-ray focusing device. Herein, we introduce a light-collecting device that uses a polycapillary x-ray lens to focus soft x-rays. After installing this lens, we confirmed that focused x-rays and a higher-intensity beam were achieved. We conclude that focusing x-rays using a polycapillary lens in the soft x-ray region is an effective method for obtaining better spectral statistics in fluorescence XAFS measurements.

[DOI: $10.1380 /$ ejssnt.2016.35]
\end{abstract}

Keywords: Fluorescence XAFS; Polycapillary x-ray lens; Synchrotron radiation; Operando conditions

\section{INTRODUCTION}

Capillary lenses have been widely used in fluorescence $\mathrm{x}$-ray analysis and $\mathrm{x}$-ray imaging analysis [1-3]. Capillary optics is a well-known x-ray focusing tool, and various capillary-based $\mathrm{x}$-ray optics have been studied to produce intense x-ray beams [4-7]. A polycapillary x-ray lens is a type of x-ray focusing optical element, which consists of several hundred thousand glass capillary tubes that focus $\mathrm{x}$-rays by total reflection within the capillaries.

The development of new approaches for facilitating insitu analysis of solutions is one of the most important problems in understanding the mechanisms of reactions at solid-liquid interfaces; in particular, light elements play an important role in the properties of environmental samples, modern materials, and functional devices in the soft x-ray region. The distinction between hard and

\footnotetext{
* This paper was presented at the 7th International Symposium on Surface Science, Shimane Prefectural Convention Center $(\mathrm{Ku}-$ nibiki Messe), Matsue, Japan, November 2-6, 2014.

$\dagger$ Corresponding author: honda.mitsunori@jaea.go.jp
}

soft x-rays is not well defined. Hard x-rays typically have energies greater than approximately $10 \mathrm{keV}$. Therefore, herein, we consider x-rays in the $1800-5000$ eV energy range (hereinafter referred to as soft x-rays) served by crystal monochromators. X-ray adsorption fine structure (XAFS) in the soft $\mathrm{x}$-ray region is a beneficial technique for studying molecular structures in both atmosphere and solutions (ambient conditions).

X-ray beam intensity can be improved using an x-ray lens. However, it can be difficult to focus an x-ray using a lens under ambient conditions, especially in the soft $\mathrm{x}$ ray region. Moreover, because attenuation of soft x-rays under ambient conditions is higher than that of hard $\mathrm{x}$ rays, good spectral statistics are not obtained.

We developed a fluorescence XAFS system toward operando conditions using soft $\mathrm{x}$-ray radiation(1.8$6.0 \mathrm{keV}$ ) from KEK-PF BL-27A [8]. However, given the large diameter of the beam of this beam line at BL-27A, it was difficult to uniformly irradiate a given sample surface. Therefore, it was necessary to improve the intensity and uniformity of the x-ray irradiation over the sample. To collimate the x-ray beam and improve its intensity, we installed an x-ray focusing device. 


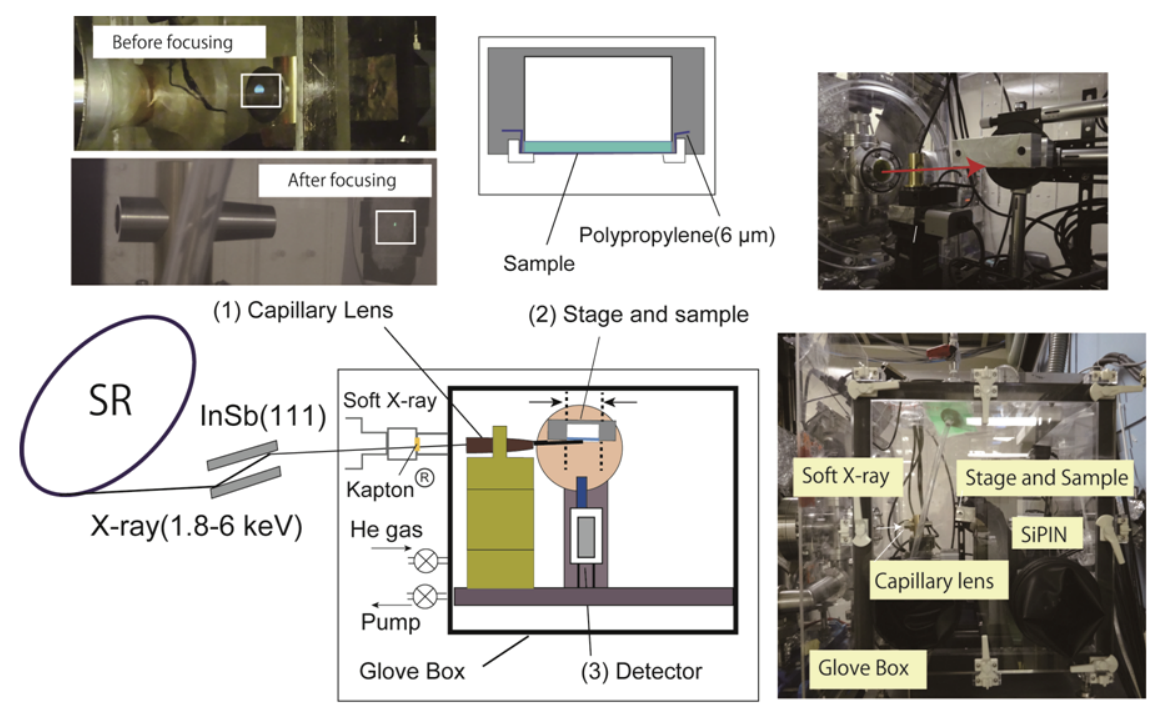

FIG. 1. Schematic diagram (left) and photograph (right) of soft x-ray XAFS system at KEK-PF BL-27A end station.

Herein, we introduce a light-collecting device that uses a polycapillary lens to focus soft x-rays. By installing this lens, we focused and improved the intensity of the x-ray beam. Furthermore, we present the results of soft x-ray focusing using a polycapillary lens along with fluorescence XAFS results obtained using the resulting system.

\section{EXPERIMENTAL}

Figure 1 shows a schematic diagram (left) and photograph (right) of the soft x-ray XAFS instrument at the end station of KEK-PF BL27A. In this beam line, $\mathrm{x}-$ rays are condensed using a mirror placed horizontal to the bending magnet, and the synchrotron radiation beam is tilted upward by $1.6^{\circ}$. The line is equipped with an InSb (111) double crystal monochromator, and the incident x-ray radiation ranges from 1800 to $5000 \mathrm{eV}$ in the soft x-ray region.

The developed fluorescence XAFS measurement system using soft $\mathrm{x}$-ray radiation consists of the following components: (1) an x-ray inlet glovebox, (2) a sample holder, and (3) a fluorescence x-ray detection unit. (1) is a vacuum-type glovebox comprising acrylic panels (Art Kagaku Co., Ltd.). The system is equipped with a gas inlet and exhaust lines, and low vacuum is achieved using a scroll pump. After evacuation for a certain period using the scroll pump, He is pumped in as a replacement gas, allowing for efficient gas replacement. Thereafter, we can perform measurements at atmospheric pressure. (2) is a sample-holding mechanism equipped with a remote-controlled stage (Sigma Koki Ltd.). The sample is mounted on the four-axis stage in the $x$ (beam direction), $y$ (perpendicular to horizontal beam), $z$ (vertical), and theta (incident angle of x-ray) directions for alignment. (3) is a small Peltier element-type Si semiconductor xray detector (X-123 SiPIN detector, Amptek Co., Ltd.), which can be installed in a small glovebox with limited space. The energy resolution of the detector is $145 \mathrm{eV}$ FWHM at $5.9 \mathrm{keV}[9]$. The detector was rotated $90^{\circ}$ rel- ative to the incident light polarization vector, i.e., it was placed directly below the sample. This allowed us to measure fluorescence XAFS spectra under conditions wherein it is possible to avoid the scattering of incident light, thus minimizing the background due to scattering as much as possible [10].

As previously explained, a polycapillary x-ray lens was developed for the synchrotron radiation system. Herein, we installed this polycapillary x-ray lens (Institute for Scientific Instruments GmbH Co., Ltd.) in a glovebox at a KEK-PF BL-27A end station. The polycapillary $\mathrm{x}$ ray lens comprises a bundle of several hundred thousand glass capillaries. The x-rays are reflected completely in the capillaries by the internal surfaces of the capillary tubes. To determine total x-ray reflectivity, several cycles of He gas replacement in the glovebox are required. If not, the x-rays are not transmitted through the capillary tubes because the tubes are filled with air in the soft $\mathrm{x}$ ray region. This prevents the scattering of incident light, thus allowing for fluorescent XAFS spectral measurement under conditions with minimal background from scattering.

The bundle of capillary tubes depends strongly on the termination of the x-ray transmission by total external reflection. Therefore, it was important to focus the x-ray beam by manipulating the x-ray lens. The ability of the capillary lens to focus the x-ray beam depends strongly on the direction $(\theta)$ and the tilt angle $(\varphi)$ of the lens with respect to the $\mathrm{x}$-ray beam.

\section{RESULTS AND DISCUSSION}

We optimized the x-ray lens position with respect to the $\mathrm{x}$-ray beam produced by the polycapillary x-ray lens. The $\mathrm{x}$-ray fluorescence intensity from the sample was recorded as the tilt angles (i.e., $\varphi$ and $\theta$ ) were varied. Figure 2 shows the x-ray lens arrangement. The orientations of the respective $\varphi$ and $\theta$ rotations are shown.

Figure 3 shows the dependency of x-ray intensity on 


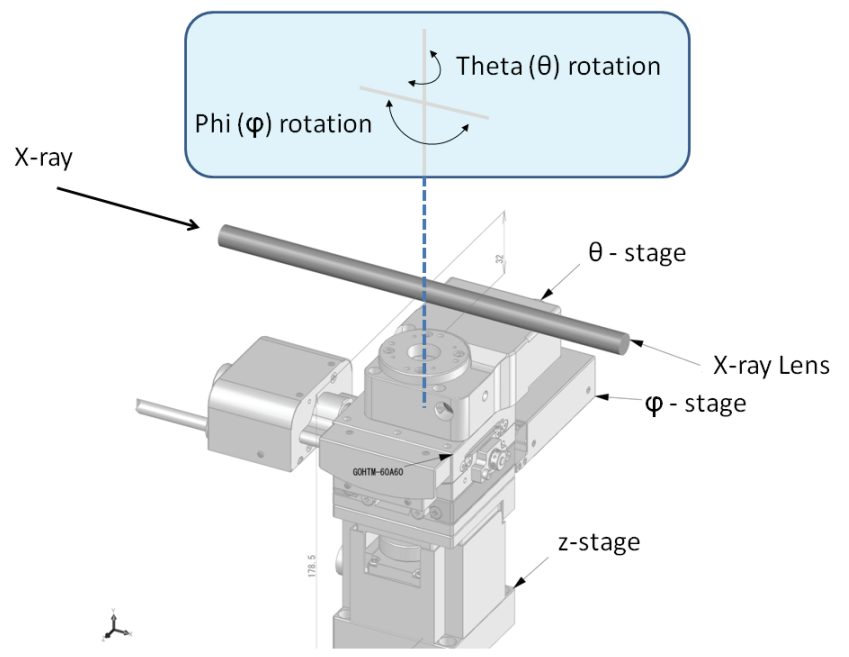

FIG. 2. Arrangements of polycapillary x-ray lens stage in a glovebox at KEK-PF BL-27A end station. Orientations of the respective $\varphi$ and $\theta$ rotations of the incident x-rays are shown.

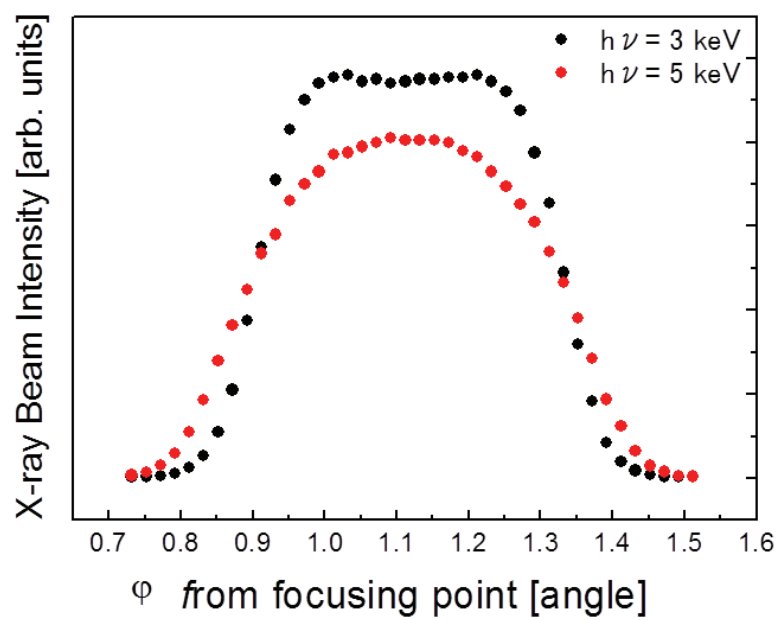

FIG. 3. Relationship between tilt angle $(\varphi)$ of polycapillary lens and x-ray beam intensity of synchrotron radiation for photon energies of 3000 (red) and $5000 \mathrm{eV}$ (black).

the tilt angle for $\mathrm{h} \nu=3000$ and $5000 \mathrm{eV}$. The intensity of synchrotron radiation x-ray profile abruptly decreased with a slight change in the tilt angle. Therefore, the tilt angle of the x-ray lens was set to values that produced the maximum intensities, as shown in Figs. 3 and 4.

Figure 3 shows the relationship between the tilt angle $(\varphi)$ of the polycapillary x-ray lens and the x-ray beam intensity of soft x-ray synchrotron radiation at 3000 (red) and $5000 \mathrm{eV}$ (black). As observed in Fig. 3, the x-ray intensity was sensitive to the tilt angle of the x-ray lens. Nearly identical characteristic profiles were obtained using the x-ray lens at both 3000 and $5000 \mathrm{eV}$. Therefore, the $\mathrm{x}$ ray lens tilt angle was fixed at the tilt angle corresponding to the maximum intensity in Fig. 3.

Figure 4 shows the relationship between the rotation angle $(\theta)$ of the polycapillary lens and the x-ray beam intensity of synchrotron radiation at $\mathrm{h} \nu=3000 \mathrm{eV}$. Moreover, the x-ray intensity was very sensitive to the rotation

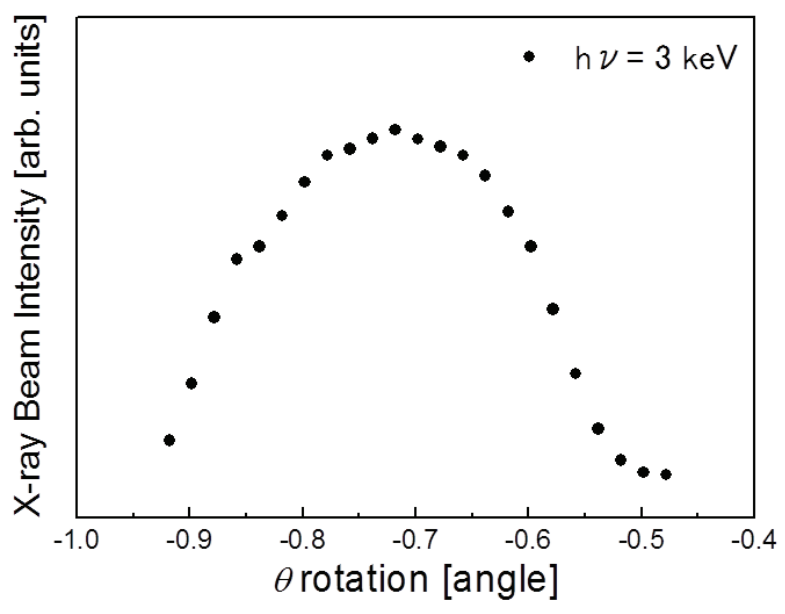

FIG. 4. Relationship between tilt angle $(\theta)$ of polycapillary lens and x-ray beam intensity of synchrotron radiation at $\mathrm{h} \nu$ $=3000 \mathrm{eV}$.

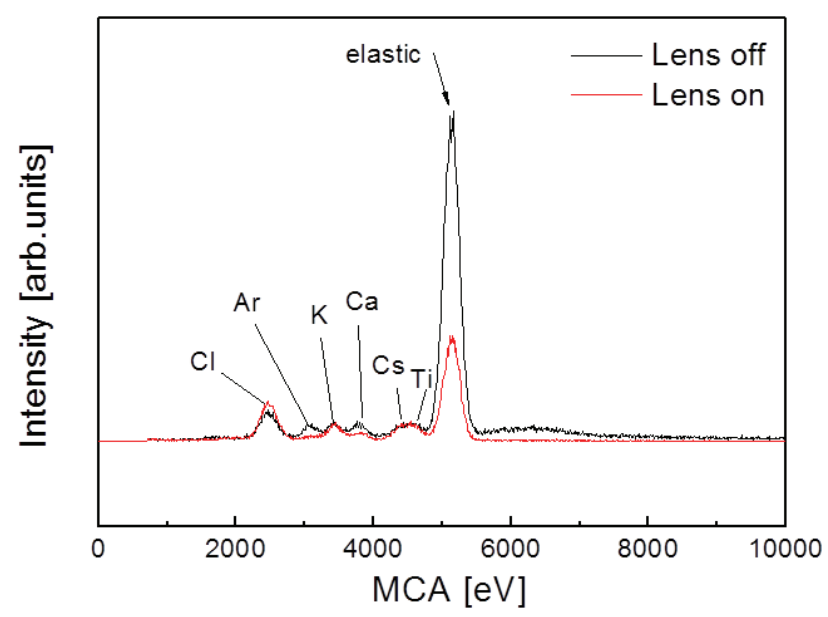

FIG. 5. MCA measurements with (red line) and without (black line) x-ray lens at $\mathrm{h} \nu=5000 \mathrm{eV}$.

angle $(\theta)$ of the $\mathrm{x}$-ray lens.

After determining the effects of the tilting angles of the $\mathrm{x}$-ray lens, to determine the energy resolution, we first recorded the multi-channel analyzer (MCA) spectra of Cssaturated vermiculite with and without the x-ray lens at $\mathrm{h} \nu=5000 \mathrm{eV}$. Figure 5 shows the MCA spectra of a Cssaturated vermiculite sample with (a) and without (b) the $\mathrm{x}$-ray lens at $\mathrm{h} \nu=5000 \mathrm{eV}$. The chemical components of vermiculite have been reported [11]. In this MCA analysis, all components were effectively detected using the x-ray lens. In the experimental photon energy region, the Cs $L_{I I I}$ edge was activated and characteristic x-rays were detected. Thus, the x-ray lens can effectively can decrease elastic scattering intensity. To measure the XAFS, the SiPIN detector was used in the multi-channel scalar mode. The spectra were obtained by detecting fluorescent $\mathrm{x}$-rays from the sample and plotting the fluorescent $\mathrm{x}$-ray intensity from the sample at each energy level. The x-ray intensity was normalized by the current aluminum mesh placed in the vacuum chamber before the sample. The NEXAFS spectra of the Cs-saturated vermiculite sam- 


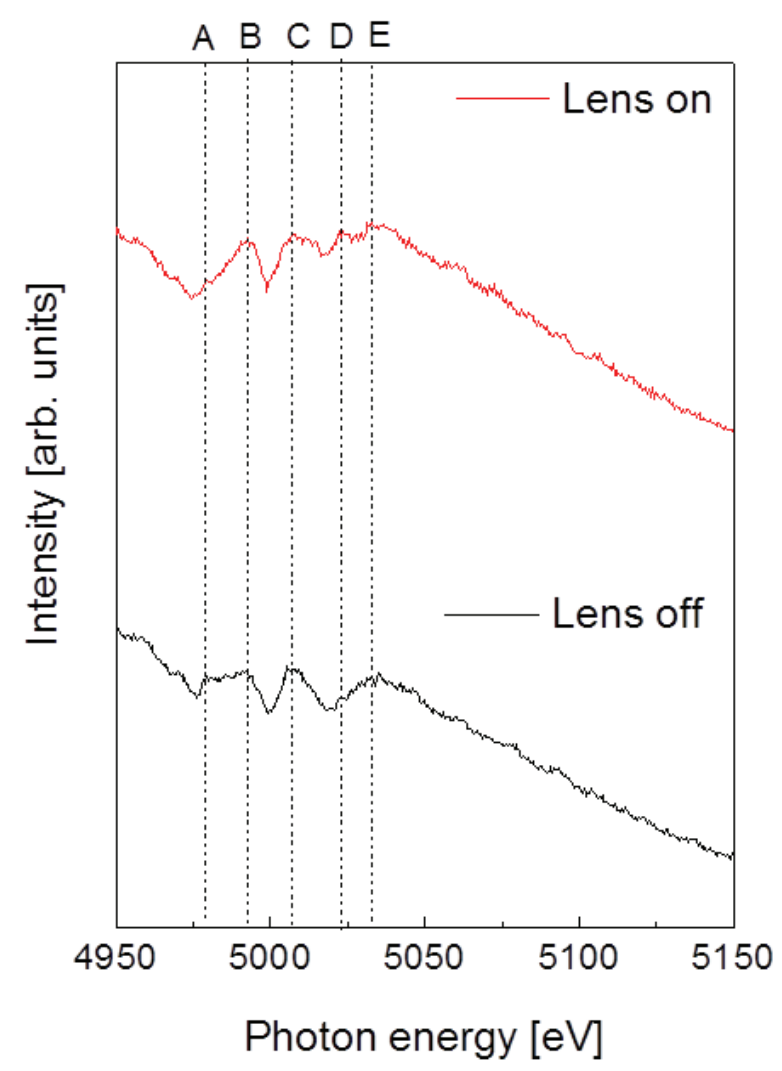

FIG. 6. Cs $L_{I I I}$-edge NEXAFS spectra of Cs-saturated vermiculite sample with (a) and without (b) x-ray lens.

ple with (a) and without (b) the x-ray lens are shown in Fig. 6. The sample was placed at the original position in a sample holder. A Cs-saturated vermiculite sample was wrapped in a thin polypropylene film $(6 \mu \mathrm{m})$ and placed in the sample holder. The measurement time was $30 \mathrm{~s}$ for each photon energy level $[\mathrm{eV}]$. We can confirm that peaks A, B, C, E are due to the Ti K-edge element in the environmental samples. When using the x-ray lens, we could observe peak D owing to the clear structure of the Cssaturated vermiculite sample in the NEXAFS spectrum at 5025-5030 eV (Fig. 6, red line). Conversely, without the X-ray lens, the detailed structure of the Cs-saturated vermiculite sample was difficult to ascertain because of the poor signal-to-background (S/B) ratio (Fig. 6, black line).

The reduction in direct x-ray elastic intensity was effective for obtaining high-S/B NEXAFS spectra to elucidate the effect of the capillary $\mathrm{x}$-ray lens. It is advantageous to employ the x-ray lens within the fluorescence XAFS system toward operando condition using soft x-rays. This technique allows for analysis of wet samples using this instrument. Moreover, we discuss our ability to focus soft $\mathrm{x}$-rays using the lens. Herein, we reported the characterization of this x-ray optical lens and its application in NEXAFS analysis. Although this x-ray lens was designed to be used at $3000 \mathrm{eV}$, it effectively performs at photon energies of 3000 and $5000 \mathrm{eV}$.

We fabricated a light-collecting device using a polycapillary lens to focus soft x-rays. The instrument was installed at a KEK-PF BL27A end station. The developed capillary lens effectively focused the x-ray beam and decreased elastic scattering intensity at 3000 and $5000 \mathrm{eV}$. This x-ray lens is applicable to XAFS measurements in the soft $\mathrm{x}$-ray region.

\section{CONCLUSIONS}

We developed an x-ray focusing system for fluorescence XAFS analysis. The developed polycapillary x-ray lens improved the spectral statistics obtained via XAFS measurements and allowed us to generate a micro-sized x-ray beam. Consequently, we could decrease elastic scattering intensity and obtain a good $\mathrm{S} / \mathrm{B}$ ratio. We expect this system will be effective in studies on environmental samples under operando conditions. Moreover, this system could be applied to the surface analyses of small regions, including the analysis of other clay minerals in aqueous environments

\section{ACKNOWLEDGMENTS}

This study was supported by KEK-PF (Proposal No. 2014G088 and 2014G118). The authors are grateful to KEK-PF staff for their support of the experiments using synchrotron radiation. This study was partially supported by a JSPS Grant-in-Aid for Young Scientists (B) 25790059 .
[1] T. Emoto, Y. Sato, Y. Konishi, X. Ding, and K. Tsuji, Spectrochim. Acta B 59, 1291 (2004).

[2] A. Bjeoumikhov, M. Erko, S. Bjeoumikhova, A. Erko, I. Snigireva, A. Snigirev, T. Wolff, I. Mantouvalou, W. Malzer, and B. Kanngießer, Nucl. Instrum. Methods Phys. Res. A 587, 458 (2008).

[3] A. G. Revenko, Spectrochim. Acta B 62, 567 (2007).

[4] Y. I. Dudchik, N. N. Kolchevsky, F. F. Komarov, Y. Kohmura, M. Awaji, Y. Suzuki, and T. Ishikawa, Nucl. Instrum. Methods Phys. Res. A 454, 512 (2000).

[5] Y. I. Dudchik, F. F. Komarov, M. a. Piestrup, C. K. Gary, H. Park, and J. T. Cremer, Spectrochim. Acta B 62, 598 (2007).
[6] T. Sun, Z. Liu, B. He, S. Wei, Y. Xie, T. Liu, T. Hu, and X. Ding, Nucl. Instrum. Methods Phys. Res. A 574, 285 (2007).

[7] S. Komatani, K. Nakamachi, K. Nakano, S. Ohzawa, H. Uchihara, A. Bando, and K. Tsuji, Nucl. Instrum. Methods Phys. Res. B 309, 260 (2013).

[8] M. Honda, Y. Baba, I. Shimoyama, and T. Sekiguchi, Rev. Sci. Instrum. 86, 035103 (2015).

[9] R. Redus, A. Huber, J. Pantazis, T. Pantazis, and D. Sperry, IEEE Nucl. Sci. Symp. Conf. Rec., 3794 (2006).

[10] D. A. Fischer, J. Colbert, and J. L. Gland, Rev. Sci. Instrum. 60, 1596 (1989).

[11] R. Motokawa, H. Endo, S. Yokoyama, T. Kobayashi, S. Suzuki, and T. Yaita, Langmuir 30, 15127 (2014). 